\title{
Extended star formation in dwarf spheroidal galaxies: The cases of Draco, Sextans, and Ursa Minor
}

\author{
C. Ikuta ${ }^{1, \star}$ and N. Arimoto ${ }^{2,3}$ \\ 1 School of Physics \& Astronomy, University of Nottingham, Nottingham, NG7 2RD, UK \\ 2 National Astronomical Observatory of Japan, 2-21-1, Osawa, Mitaka, Tokyo 181-8588, Japan \\ e-mail: arimoto@optik.mtk.nao.ac.jp \\ 3 Institute of Astronomy, University of Tokyo, 2-21-1, Osawa, Mitaka, Tokyo 181-0015, Japan
}

Received 22 October 2001 / Accepted 12 April 2002

\begin{abstract}
Star formation and chemical enrichment histories of the dwarf spheroidal galaxies (dSphs) Draco, Sextans, and Ursa Minor are investigated by means of chemical evolution models and a simulation code for colour-magnitude diagrams (CMDs). The CMD simulation code is designed to fully consider effects of the chemical evolution on stellar evolution and photometric properties. For this aim, star formation and chemical enrichment histories are calculated consistently in the code. Comparisons between the chemical evolution models and the observed abundance patterns reveal that the star formation rates were very low (1-5\% of that of the solar neighbourhood disc) and that the initial star formation continued for a long duration ( $>3.9-6.5$ Gyr) in these dSphs. This star formation history can reproduce morphologies of the observed CMDs, such as narrow red giant branches and red horizontal branches and succeeds in solving the second parameter problem of the dSph Draco. Hence, both of the abundance patterns and the morphologies of the CMDs can be explained by the star formation histories characterised by the low star formation rate and the long duration of the star formation period. Because of the low star formation rates, plenty of gas remains at the final epoch of star formation. We suggest that gas stripping by the Galaxy results in termination of star formation in the dSphs.
\end{abstract}

Key words. galaxies: dwarf - galaxies: abundances - galaxies: evolution - galaxies: Local Group - galaxies: stellar content

\section{Introduction}

Satellite dwarf spheroidal galaxies of the Milky Way have been considered to be similar to globular clusters (e.g., Hodge 1971), since they are composed of old metal-poor stars and contain little gas. Recent analyses of the colour-magnitude diagrams (CMDs), however, reveal a surprising fact that dwarf spheroidal galaxies (dSphs) are of composite stellar populations. Some of dSphs formed stars continuously and/or intermittently for many Gyr (e.g., the dSph Carina: Smecker-Hane et al. 1996; the dSph Leo I: Gallart et al. 1999). Low-resolution spectra of individual stars along the red giant branch (RGB) have shown that both Galactic and M31 satellite dSphs have internal metallicity dispersions (e.g., Suntzeff et al. 1993; Smecker-Hane \& McWilliam 1999; Côté et al. 1999). Using high dispersion ( $R=34$ 000) spectroscopy, Shetrone et al. (2001) have recently presented abundances of various elements in the three dSphs Draco, Sextans, and Ursa Minor, which are all satellite galaxies of the Milky Way. They report that the abundance ratios of $\alpha$ elements $(\mathrm{O}, \mathrm{Si}, \mathrm{Mg}$, etc.) relative to iron $([\alpha / \mathrm{Fe}])$ are nearly equal to solar at low metallicity $([\mathrm{Fe} / \mathrm{H}]<-1.4)$. This suggests

Send offprint requests to: C. Ikuta,

e-mail: chisato.ikuta@nottingham.ac.uk

* JSPS fellow. that the stars were formed in the gas enriched by Type Ia supernovae (SNe Ia) as well as by Type II supernovae (SNe II), that is, star formation in the dSphs continued over the lifetime (typically 1-2 Gyr) of SNe Ia progenitors. This result allows us to directly study the chemical enrichment histories (CEHs) of the dSphs Draco, Sextans, and Ursa Minor.

One problem in deriving an accurate SFH from stellar colours is the so-called age-metallicity degeneracy. Older stars become redder and the same is true for more metal-rich stars. Hence, young and metal-rich stars show colours similar to old and metal-poor ones. We have empirically solved the age-metallicity degeneracy for the three dSphs cited above to derive their star formation histories (SFHs) to a level of accuracy that is impossible in other galaxies in the Local Group. To reveal SFHs in detail would help to understand how large galaxies and the environments affect the evolution of dwarf galaxies.

In addition to a study of the evolution of dwarf galaxies themselves, one can also learn about the possible merging history of the Milky Way by comparing the abundances of the satellite dwarf galaxies with those of Galactic field halo stars. In a hierarchical galaxy formation scenario, larger galaxies grew at the expense of smaller gaseous fragments(e.g., White \& Rees 1978; Kauffmann et al. 1993; Blumenthal et al. 1994; 
Cole et al. 1994). From the observational perspective, Searle \& Zinn (1978) proposed a model in which the Galactic halo was formed via infall and destruction of proto-Galactic fragments. Increasing empirical and analytical evidence suggests that the Galactic halo was, at least in part, assembled from chemically-distinct, low-mass fragments (e.g., Searle \& Zinn 1978; Yanny et al. 2000). Such evidence includes the recent discovery of the tidally disturbed dSph Sagittarius which is falling onto the Galaxy (Ibata et al. 1994) as well as the numerous reports of kinematical substructure among halo field stars (e.g., Chen 1998). The scenario can be tested by comparing abundance patterns of field stars in the Galactic halo and those of satellite dwarf galaxies. If today's dwarf galaxies in the Local Group were the counterparts of proto-Galactic gaseous fragments, the abundance patterns of dwarf galaxies should be similar to those of field stars in the Galactic halo. The observations by Shetrone et al. (2001), however, have shown that the patterns of abundance ratio in the dSphs are different from those of the Galactic halo stars. The dSphs have $-0.4 \leq[\mathrm{Mg} / \mathrm{Fe}] \leq 0.4$, while field stars in the Galactic halo have $0.2 \leq[\mathrm{Mg} / \mathrm{Fe}] \leq 0.6$. The authors concluded that the Galactic halo was unlikely to have formed via the accretion of objects similar to the dSphs Draco, Sextans, and Ursa Minor.

In this paper, we discuss the SFHs and the CEHs of the three dSphs Draco, Sextans, and Ursa Minor. Using the abundance patterns and the CMDs, we show that the low (1-5\% of the solar neighbourhood) star formation rate and relatively long duration (3.9-6.5 Gyr) of initial star formation explain both the abundance patterns and morphologies of CMDs such as wellpopulated red horizontal branch (RHB) and the tight red giant branch (RGB).

The paper is organised as follows. In Sect. 2, chemical evolution model is confronted with the observed stellar abundances to study the CEHs and SFHs. In Sect. 3, by calculating CMDs by using our CMD simulation code (Ikuta 2001), we will show that the SFHs derived in the previous section succeed in reproducing observed CMDs. Discussions and conclusions are presented in Sects. 4 and 5, respectively.

\section{Observed abundances and chemical evolution}

\subsection{Chemical evolution model}

Generally stellar birth rate is separated into two independent functions. The birth rate of stars with mass between $m$ and $m+$ $\mathrm{d} m$ is described as $C(t) \phi(m) \mathrm{d} m$, where $C(t)$ and $\phi(m)$ are the $S F R$ and the initial mass function (IMF), respectively (Tinsley 1980). The IMF is assumed to be time invariant with a powerlaw spectrum. Normalising to unity, we have

$\phi(m)=\frac{(x-1) m_{1}^{x-1}}{1-\left(m_{1} / m_{\mathrm{u}}\right)^{x-1}} m^{-x}$,

where the lower and the upper mass limits are assumed to be $m_{1}=0.1 M_{\odot}$ and $m_{\mathrm{u}}=60 M_{\odot}$, respectively. The Salpeter IMF has $x=1.35$ in this definition. Similarly to the solar neighbourhood disc model of chemical evolution (Arimoto et al. 1992), the Salpeter IMF is assumed in this section. Models with

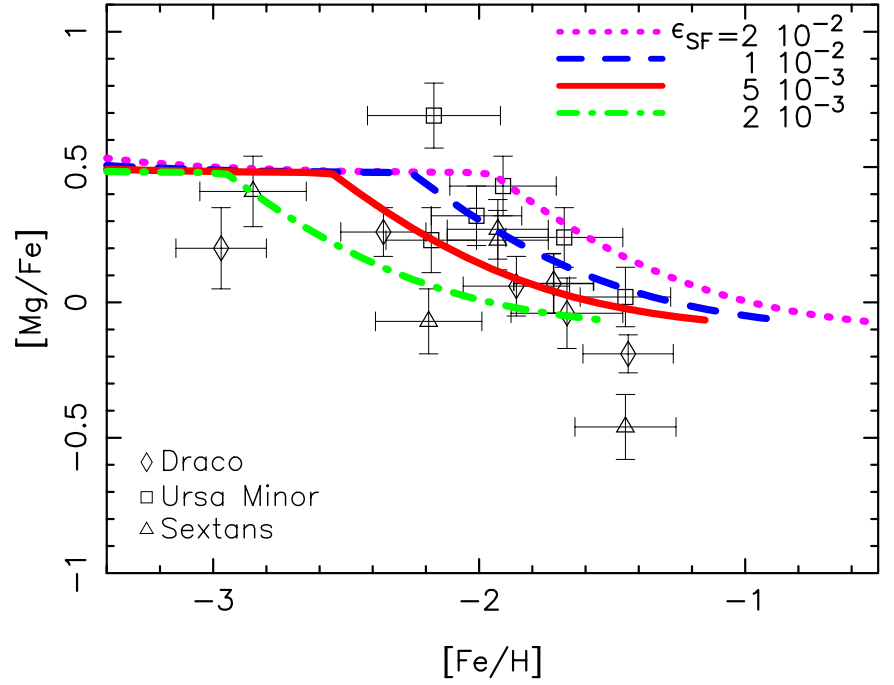

Fig. 1. Theoretical abundance patterns together with the observations (Shetrone et al. 2001) for stars in the dSphs Draco, Sextans, and Ursa Minor. The meaning of the marks is written on the panel. The same IMF (the Salpeter IMF: $x=1.35)$ with upper $\left(m_{\mathrm{u}}=60 M_{\odot}\right)$ and lower $\left(m_{l}=0.1 M_{\odot}\right)$ mass limits are adopted in all the model, while different values of $\epsilon_{\mathrm{SF}}\left(\mathrm{Gyr}^{-1}\right)$ are assumed; model A $\left(\epsilon_{\mathrm{SF}}=2 \times 10^{-2} \mathrm{Gyr}^{-1}\right.$ : dotted line $)$; model $\mathrm{B}\left(\epsilon_{\mathrm{SF}}=1 \times 10^{-2} \mathrm{Gyr}^{-1}\right.$ : dashed line $)$; model $\mathrm{C}$ $\left(\epsilon_{\mathrm{SF}}=5 \times 10^{-3} \mathrm{Gyr}^{-1}\right.$ : solid line $)$; and model D $\left(\epsilon_{\mathrm{SF}}=2 \times 10^{-3} \mathrm{Gyr}^{-1}\right.$ : dotted-dashed line). (This figure is available in color in electronic form.)

different IMFs will be discussed in Sect. 4. The gas mass $M_{\mathrm{g}}(t)$ changes through star formation:

$\frac{\mathrm{d} M_{\mathrm{g}}(t)}{\mathrm{d} t}=-C(t)+E(t)$

where $E(t)$ is the gas ejection rate from dying stars, assuming a closed-box model. In the case of the Galactic disc, an infall model is generally assumed to solve the so-called G-dwarf problem (e.g., Tinsley 1980; Arimoto et al. 1992). However, there is no observational evidence for the G-dwarf problem in dwarf galaxies, thus we do not consider the infall of gas here. Gas outflow during the star formation is not considered, either. A recent numerical experiment showed that the fraction of mass loss from dwarf galaxies is small (at most $\sim 7$ percent of the galaxy mass; MacLow \& Ferrara 1999). Metal-enhanced wind is often introduced to explain observed properties of dwarf galaxies (e.g., Vader 1986). MacLow \& Ferrara (1999) showed that indeed most of the metals are able to leave dwarf galaxies during star burst, although the mass loss rate is small. Virtually no metals are retained in dwarf galaxies if high energy is supplied by supernova explosions (see Table 3 in their literature for details). Complete metal loss means no chemical enrichment in dwarf galaxies. This is inconsistent with observations which show clearly that dSphs are chemically polluted. Their results suggest that only star formation of a low rate allows a dwarf galaxy to retain a large fraction of heavy elements produced in massive stars. As will be shown later, very low $S F R$ s are indeed required to explain the observed abundances and CMDs. Thus, a simple model is adopted here and a galaxy is assumed to be a closed system. 

$f_{\mathrm{g}}(t)$ :

The $S F R$ is assumed to be proportional to the gas fraction

$C(t)=\epsilon_{\mathrm{SF}} M_{\mathrm{G}} \cdot f_{\mathrm{g}}(t)=\epsilon_{\mathrm{SF}} \cdot M_{\mathrm{g}}(t)$

with

$f_{\mathrm{g}}(t) \equiv M_{\mathrm{g}}(t) / M_{\mathrm{G}}$,

where $\epsilon_{\mathrm{SF}}^{-1}(\mathrm{Gyr})$ and $M_{\mathrm{G}}$ are the timescale of star formation and the initial total mass of a galaxy, respectively.

The evolution of the abundance of the $i$ th element $Z_{i}(t)$ is given as

$\frac{\mathrm{d}\left(Z_{i} M_{\mathrm{g}}\right)}{\mathrm{d} t}=-Z_{i} C(t)+E_{Z_{i}}(t)+Z_{i}(0) M_{\mathrm{G}}$,

where $E_{Z_{i}}(t)$ is the total ejection rate of processed and unprocessed $i$ th element, $Z_{i}(0)$ is the initial abundance of $i$ th element in a proto-cloud. Hereafter, $Z_{i}(0)=0$ is always assumed, i.e., a proto-cloud was metal-free. We calculate $E(t)$ and $E_{Z_{i}}(t)$ from the following integrals:

$E(t)=\int_{m_{\mathrm{t}}}^{m_{\mathrm{u}}}\left(1-w_{m}\right) C\left(t-t_{m}\right) \phi(m) \mathrm{d} m$,

$$
\begin{aligned}
& E_{Z_{i}}(t)= \\
& \quad \int_{m_{\mathrm{t}}}^{m_{\mathrm{u}}}\left[\left(1-w_{m}\right) Z_{i}\left(t-t_{m}\right)+p_{i m}\right] C\left(t-t_{m}\right) \phi(m) \mathrm{d} m,
\end{aligned}
$$

where $t_{m}$ is the lifetime of a star with mass $m$ and the lower limit $m_{t}$ is the stellar mass with lifetime $t_{m}=t$. Nucleosynthesis data $w_{m}$ and $p_{\text {im }}$ are the remnant mass fraction and the total mass fraction of processed and unprocessed $i$ th element. It is still debated whether stellar metallicity affects both $w_{m}$ and $p_{i m}$, but no firm consensus has been reached yet. Thus we neglect these possible effects: instead we adopt the values calculated for the solar abundance. We assume that the lower mass limit $m_{1, \text { II }}$ for a progenitor of Type II supernova (SNeII) is $10 M_{\odot}$ (Tsujimoto et al. 1995). Nucleosynthesis of SNeII and of SNeIa are explicitly included, since the former profoundly produce the $\alpha$ elements (e.g., $\mathrm{O}, \mathrm{Mg}, \mathrm{Si}, \mathrm{Ca}$ ), while the latter the iron-peak elements (e.g., Fe, Ni, Co). The data of nucleosynthesis are taken from Thielemann et al. (1996) for SNeII, Nomoto et al. (1996) for SNeIa, and Renzini \& Voli (1981) for low-mass $\left(\leq 8 M_{\odot}\right)$ stars. Progenitors of SNe Ia are generally considered to have longer lifetimes than those of SNe II, since a majority of them are likely to be Chandrasekhar mass white dwarfs (e.g., Nomoto et al. 1997). Observations for disc (Edvardsson et al. 1993) and halo (e.g., McWilliams et al. 1995; Gratton \& Sneden 1987) stars in the Milky Way show that there is a sudden decline of $[\alpha / \mathrm{Fe}]$ in the $[\alpha / \mathrm{Fe}]-[\mathrm{Fe} / \mathrm{H}]$ diagram. The decline is generally interpreted as an onset of SN Ia explosions. Studying observed $[\mathrm{O} / \mathrm{Fe}]$ break at $[\mathrm{Fe} / \mathrm{H}] \sim-1$ for disc and halo stars in the Milky Way, Yoshii et al. (1996) suggested that the effective lifetime of SNe Ia is around 1.5 Gyr. A new SNIa model (Hachisu et al. 1999) suggested that occurrence of SNeIa depends on the metallicities of progenitors as well as the lifetimes. Based on the new SN Ia model, Kobayashi et al. (1998) predicted that SN Ia events occur only for the progenitors with
Table 1. Model parameters.

\begin{tabular}{cccc}
\hline \hline Model & $x$ & $\epsilon_{\mathrm{SF}}\left(\mathrm{Gyr}^{-1}\right)$ & $\Delta T_{\mathrm{SF}}(\mathrm{Gyr})$ \\
\hline $\mathrm{A}$ & 1.35 & $2 \times 10^{-2}$ & 2.5 \\
$\mathrm{~B}$ & 1.35 & $1 \times 10^{-2}$ & 3.9 \\
$\mathrm{C}$ & 1.35 & $5 \times 10^{-3}$ & 6.5 \\
$\mathrm{D}$ & 1.35 & $2 \times 10^{-3}$ & 12 \\
$\mathrm{E}$ & 1.75 & $2 \times 10^{-1}$ & 1.6 \\
$\mathrm{~F}$ & 1.95 & $2 \times 10^{-1}$ & 1.8 \\
$\mathrm{G}$ & 2.15 & $2 \times 10^{-1}$ & 2.2 \\
\hline
\end{tabular}

$[\mathrm{Fe} / \mathrm{H}] \geq-1.1$. On the observational side, $\alpha$ element to iron abundance ratios of the three dSphs, Draco, Sextans, and Ursa Minor (Shetrone et al. 2001) have indicated a significant contribution from SNeIa at a metallicity lower than $[\mathrm{Fe} / \mathrm{H}]=-1.0$. The observed trends of $[\alpha / \mathrm{Fe}]$ are difficult to explain by the new SNIa model. Thus, the metallicity effect on SNeIa is not considered here and the lifetime of the progenitors is assumed to be 1.5 Gyr (Yoshii et al. 1996).

\subsection{Comparison with the observation}

Figure 1 reproduces the observed stellar abundances of the $\mathrm{dSphs}$ Draco, Sextans, and Ursa Minor in the $[\mathrm{Mg} / \mathrm{Fe}]-[\mathrm{Fe} / \mathrm{H}]$ diagram (Shetrone et al. 2001). Despite the narrow widths of the RGB (see Fig. 1 of Shetrone et al. 2001), the iron abundances exhibit a large dispersion $(-3 \leq[\mathrm{Fe} / \mathrm{H}] \leq-1.4)$, and the abundance ratios of the $\alpha$-elements (e.g., $\mathrm{Mg}$ ) to iron are near or below solar. The abundance ratio $[\mathrm{Mg} / \mathrm{Fe}]$ starts to decrease at $[\mathrm{Fe} / \mathrm{H}] \simeq-2$. On the other hand, $[\mathrm{Mg} / \mathrm{Fe}]$ in the solar neighbourhood decreases with $[\mathrm{Fe} / \mathrm{H}]$ at around $[\mathrm{Fe} / \mathrm{H}] \geq-1$, which is generally interpreted as the onset of SNeIa explosions (e.g., Matteucci \& Greggio 1986). Supposing that the decline of $[\mathrm{Mg} / \mathrm{Fe}]$ in the dSphs is also caused by SNeIa, the star formation should have continued longer than the lifetime (typically 1-2 Gyr) of progenitors of SNeIa and the SFRs should be much lower than that of the solar neighbourhood. A time-delay model (Matteucci \& Brocato 1990) of the SNeIa precisely predicted that the low star formation rate results in lower $[\mathrm{Mg} / \mathrm{Fe}]$ ratios relative to stars in the Galaxy.

In Fig. 1, theoretical abundance patterns of our models $\mathrm{A}-\mathrm{D}$ are superposed. The models assume different $\epsilon_{\mathrm{SF}}$ of $2 \times 10^{-2}, 1 \times 10^{-2}, 2 \times 10^{-3}, 1 \times 10^{-3}\left(\mathrm{Gyr}^{-1}\right)$ as indicated in Fig. 1 . The model parameters are summarised in Table 1 . A typical value of $\epsilon_{\mathrm{SF}}$ for the solar neighbourhood disc is $\epsilon_{\mathrm{SF}}=0.2 \mathrm{Gyr}^{-1}$ (Arimoto et al. 1992).

The models B-D shown in Fig. 1 give a good fit to the observed abundance patterns. Since the abundance ratios resulting from the model A are too high to reproduce the observed ones, the model $\mathrm{A}$ is rejected. The model D with $\epsilon_{\mathrm{SF}}=1 \times 10^{-3} \mathrm{Gyr}^{-1}$ is not appropriate, either. Since chemical evolution is so slow, it takes around $15 \mathrm{Gyr}$ to reach $[\mathrm{Fe} / \mathrm{H}]=-1.4$, which is the highest abundance observed in these galaxies. If this model is adopted, the star formation must continue till now. Clearly this is inconsistent with the fact that no star formation occurs currently in these galaxies. Thus, only models $\mathrm{B}$ and $\mathrm{C}$ remain as 


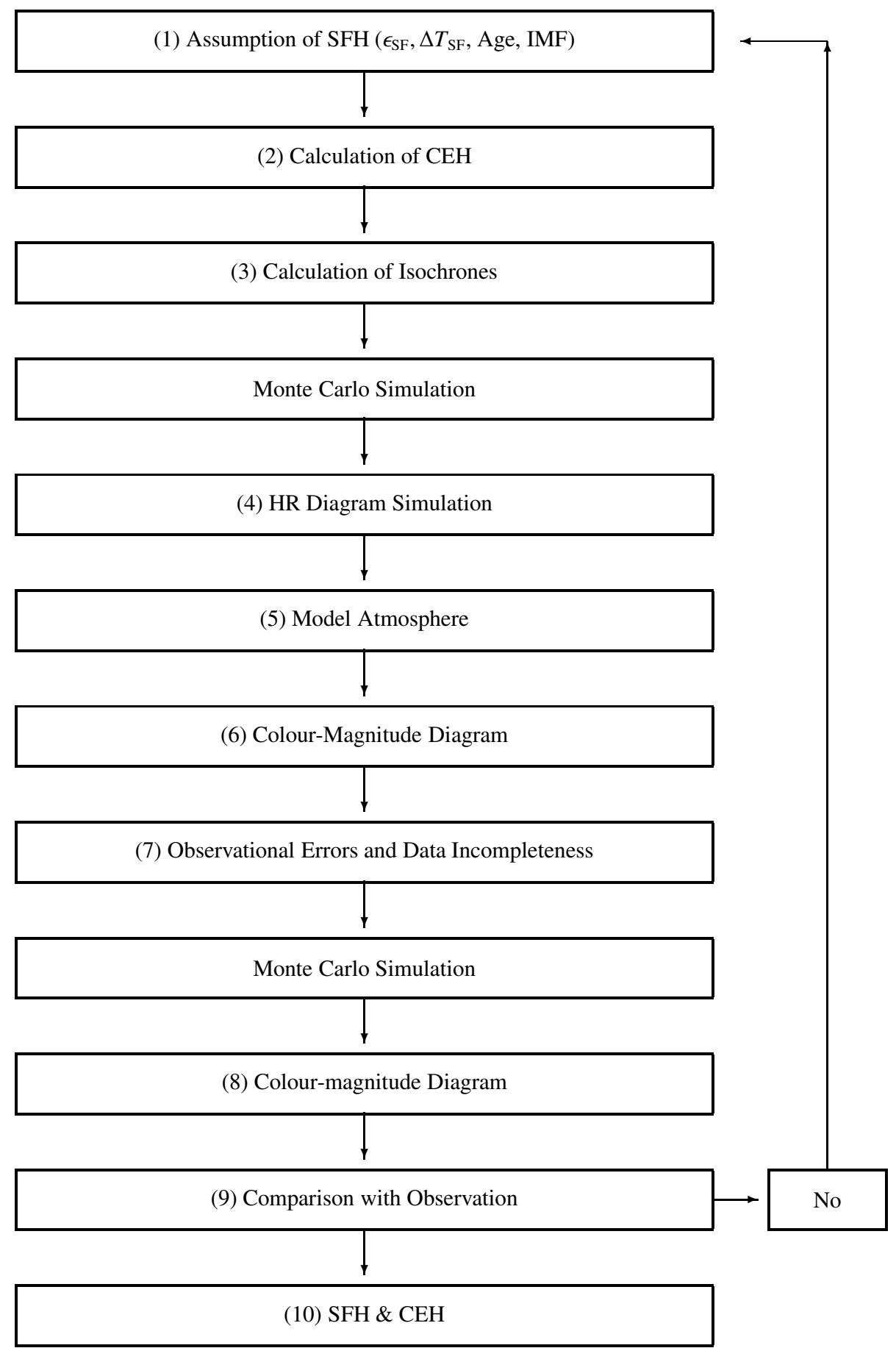

Fig. 2. Flow-chart of a CMD simulation code.

being acceptable. We note that the observational data for the $\mathrm{dSph}$ Sextans have lower signal-to-noise ratios than those for the dSphs Draco and Ursa Minor (see Table 2 of Shetrone et al. 2001) and the abundances of the dSph Sextans are less reliable than those of the other two dSphs. Thus, it is not necessary to take the discrepancy between the model predictions and the abundances of the dSph Sextans too seriously.

In short, the abundance patterns of the dSphs Draco, Sextans, and Ursa Minor all suggest that the chemical enrichment occurred with $S F R$ s which are much lower than normal spiral galaxies (only 1-5\%).

\section{CMDs}

The models $\mathrm{B}$ and $\mathrm{C}$ fit well to the observed trends in the $[\mathrm{Mg} / \mathrm{Fe}]-[\mathrm{Fe} / \mathrm{H}]$ diagram. Next, we discuss if the colourmagnitude diagrams (CMDs) predicted by these models can reproduce the observed ones. The CMDs provide us with the 


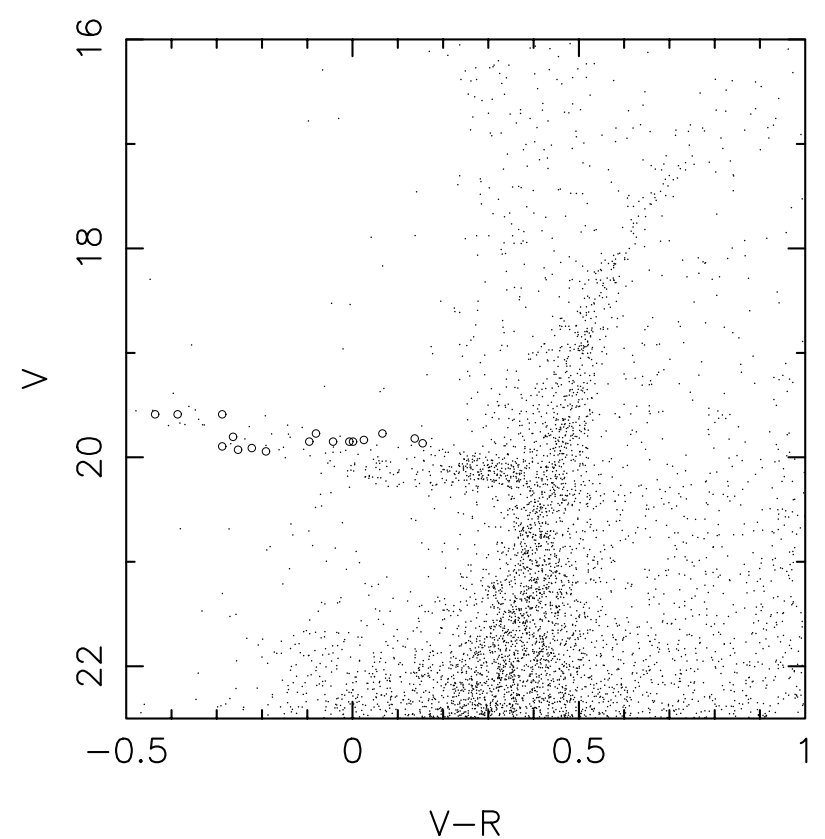

Fig. 3. CMD of the C0 region of the Draco dSph observed by Piatek et al. (2001). The points marked with open circles correspond to some confirmed RR Lyrae stars (Baade \& Swope 1961).

most detailed information to follow SFHs and CEHs back to the oldest stars. The SFH derived from the abundance patterns in the previous section must give a consistent CMD with the observations.

\subsection{Simulation code of a CMD}

Simulating numerical CMDs has become a standard technique to study the SFHs of nearby galaxies through the observed CMDs. Galaxies are composed of complex stellar populations, and the observed CMDs are affected by photometric errors and detection incompleteness. A Monte Carlo simulation allows us to take into account both simultaneously. A composite stellar population is randomly generated according to predictions of stellar evolution theory with the IMF and SFR being assumed, and then uncertainties of the data such as the increasing scatters and rising incompleteness at fainter magnitudes are taken into account.

Because of these advantages, this approach is becoming more and more frequently used to study the stellar populations of nearby galaxies. The approach was first applied by Ferraro et al. (1989) and was fully described by Tosi (1991) and Greggio et al. (1993). A more quantitative method, the $R$ method, to compare simulated and observed CMDs was presented by Bertelli et al. (1992) and was described by Vallenari et al. (1996) in more detail. The statistical comparison was further developed by Tolstoy \& Saha (1996) and Tolstoy (1996) by adopting Bayes' theorem. Now several groups have constructed CMD simulators and have investigated SFHs of galaxies in the Local Group (e.g., Aparicio et al. 1996; Dolphin 1997; Hernandez et al. 1999; Gallart et al. 1999).

We should point out, however, that there were two problems in the previous studies. First, all the approaches so far developed use the so-called optimising method of stellar population synthesis. The best mixture of stellar population is searched iteratively to reproduce the CMDs. Problems with this approach are the unproven uniqueness of the solution (e.g., Greggio et al. 1999) and a lack of evolutionary information.

Second, stellar metallicity was assumed a priori in all the simulation codes presented previously. Some introduced metallicity variation in time (e.g., Gallart et al. 1999), but the variation assumed was independent of the SFH. Since the metallicity affects evolutionary tracks and atmospheres of stars, the colours and luminosities of stars are changed by metallicity. Thus, it is crucial to use a simulator of CMDs which fully takes into account a CEH to interpret the CMDs properly and to derive the SFHs accurately. The simplification and/or neglect of chemical evolution lead to a serious problem called the age-metallicity degeneracy. Since stellar colours become bluer when stars are younger and/or poorer in metallicity, there are at least two interpretations for a given position of a star in the CMD; young and metal-rich or old and metal-poor. Therefore, an assumption of the metallicity (or age) of a certain stellar population may result in wrong estimation of age or metallicity, due to the age-metallicity degeneracy.

Aiming to solve the degeneracy, we have built a numerical simulation code of CMD morphology (Ikuta 2001). In the code, we adopt an evolutionary method of stellar population synthesis (e.g., Arimoto \& Yoshii 1986). A galaxy is assumed to have been a proto-galactic gas cloud at the beginning. Stars formed and newly processed elements were ejected from stars at the end of their lives either via stellar wind or via supernova explosions (SNeIa and SNeII). The gas was chemically enriched and the next generation of stars changed their evolution and photometric properties due to a metallicity increase. The code is particularly designed to disentangle stellar age and metallicity both of which heavily affect the morphology of CMDs. To take into account effects of chemical evolution, a fine interpolation in stellar ages and metallicities is adopted in our code. The later stages of stellar evolution, in particular, horizontal branch (HB), asymptotic giant branch (AGB), and post-AGB stars are treated in a sophisticated way, since these bright stars are crucial for this purpose. In this section, the procedure of our Monte Carlo simulation of CMDs and the ingredients are detailed.

\subsubsection{Outline of the simulation procedure}

Figure 2 shows the flow-chart of the simulation. First, a SFH is assumed. The parameters to describe the SFH are the timescale of star formation $\epsilon_{\mathrm{SF}}^{-1}(\mathrm{Gyr})$, the duration of star formation $\Delta T_{\mathrm{SF}}$ (Gyr), the slope of the IMF $(x)$, and the age of a galaxy. Second, chemical evolution is calculated, which gives the total number of stars at each metallicity at each time step. Third, a HR diagram is calculated by using stellar evolutionary tracks, where a Monte Carlo simulation is performed to assign stellar masses. Fourth, the effective temperature $T_{\text {eff }}$ and luminosity $L$ of a star are transformed to colour and magnitude by using a library of stellar model atmospheres. Fifth, a Monte Carlo simulation is performed again to take into account observational errors and detection completeness, and thus the CMD is obtained. A number of stars in the simulated CMD is adjusted to an observed 

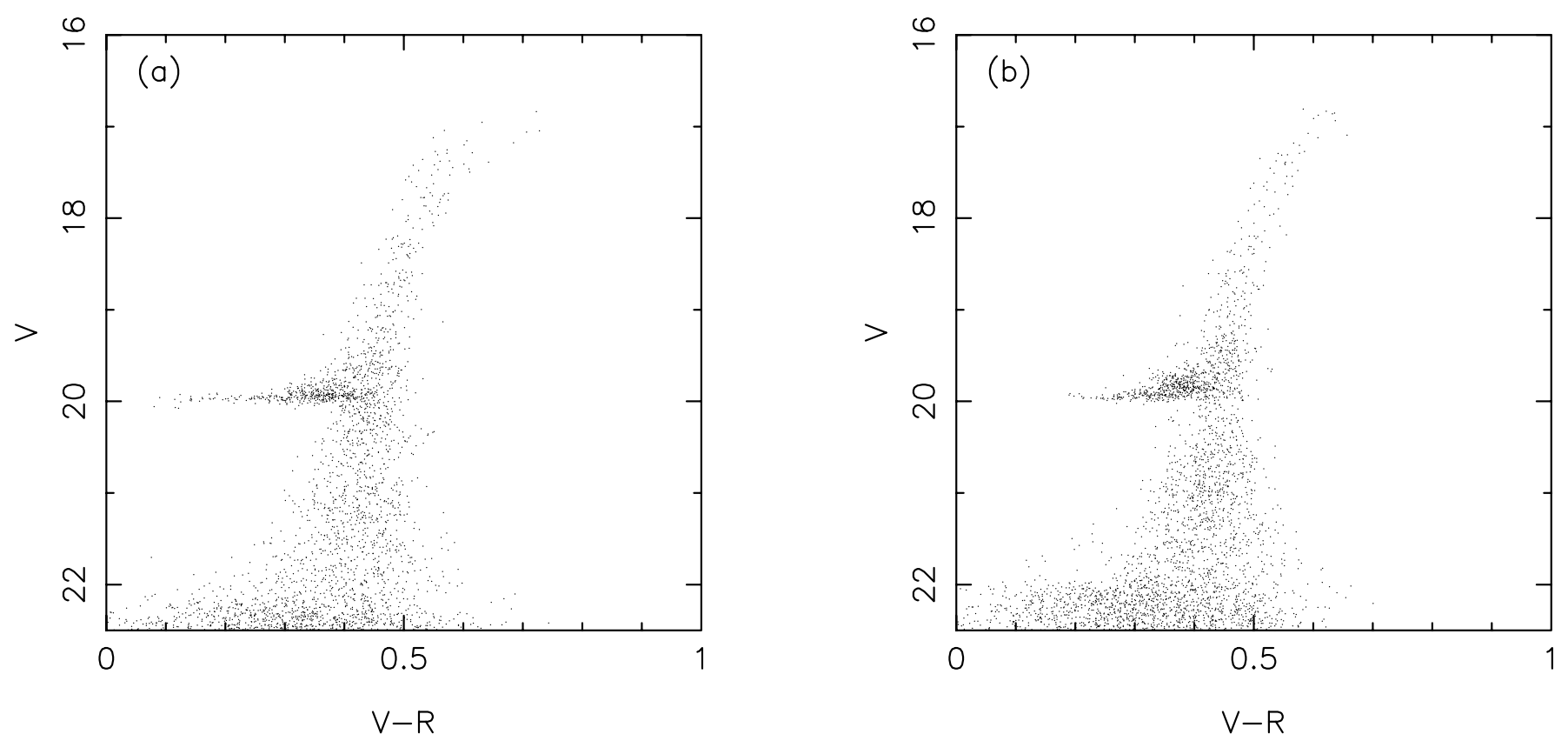

Fig. 4. Simulated CMDs of models B (left) and C (right). In model $\mathrm{B}, \epsilon_{\mathrm{SF}}=1 \times 10^{-2}\left(\mathrm{Gyr}^{-1}\right)$ and $\Delta T=3.9 \mathrm{Gyr}$ are assumed, while $\epsilon_{\mathrm{SF}}=$ $5 \times 10^{-3}\left(\mathrm{Gyr}^{-1}\right)$ and $\Delta T=6.5 \mathrm{Gyr}$ are adopted in model C. In the both models, we assumed the same upper and lower mass limits $\left(m_{\mathrm{u}}=60 M_{\odot}\right.$ and $m_{1}=0.1 M_{\odot}$, respectively), the Salpeter IMF, and the galactic age of $12 \mathrm{Gyr}$. Observational errors and data incompleteness are taken from Piatek et al. (2001).

CMD. Finally, the simulated CMD is confronted with the observed one by visual inspection. If they are inconsistent, the parameters of the SFH are changed, and the simulation is restarted. If the simulated CMD fits the observation, the SFH and $\mathrm{CEH}$ are derived. Ingredients of the simulation, such as stellar evolutionary tracks, model atmospheres, and observational conditions, are described in the subsequent section.

\subsubsection{Stellar tracks and model stellar atmosphere}

Stellar evolutionary tracks give luminosities, effective temperatures, and surface gravities of stars with given mass and chemical composition as a function of age. The tracks depend on the basic parameters such as the initial mass and chemical compositions. The properties of stellar populations in galaxies also depend on other properties not explicitly included in most of current stellar evolution models, i.e., the stellar rotation and close binary companions. The numerical calculations are also sensitive to the treatment of the convection, such as a mixing length parameter $l / H_{\mathrm{p}}$ and convective overshooting.

The Padova stellar evolutionary tracks are adopted for stars from the main sequence (MS) to the early asymptotic giant branch (EAGB) stars. The Padova tracks cover wide ranges in age ( 0 to $16 \mathrm{Gyr})$ and metallicity $(Z=0.0001$ to $Z=0.05)$, and are one of the most complete sets of stellar evolutionary models currently available. The Padova tracks were calculated with revised radiative opacities (Iglesias et al. 1992) and with $l / H_{\mathrm{p}}=$ 1.63. The adopted tracks were calculated for the following set of chemical compositions: $(Y=0.230, Z=0.0001$; Girardi et al. 1996), $(Y=0.230, Z=0.0004$; Fagotto et al. 1994a), $(Y=0.240, Z=0.004$; Fagotto et al. 1994b), $(Y=0.250$,
$Z=0.008$; Fagotto et al. 1994b), $(Y=0.280, Z=0.02$; Bressan et al. 1993), and ( $Y=0.352, Z=0.05$; Fagotto 1994a).

To obtain the isochrone for a given metallicity, the original isochrones are linearly interpolated in metallicity. Since the metallicity changes stellar evolution non-linearly, any extrapolation in metallicity should be avoided. Thus, stars of metallicity lower $(Z<0.0001)$ and higher $(Z>0.05)$ than the Padova tracks do not appear in our simulated CMDs and are not considered here. This is justified, because no significant populations of such extreme metallicities are known to exist in dwarf galaxies. In addition to these, close binary systems are not included, since the input tracks are only for isolated single stars. Although our code does not explicitly include binary stars, it effectively takes into account apparent binary stars as stellar blends. Gallart et al. (1999) reported a study that is more detailed on this issue.

For the RGB evolution, the mass loss law of Reimers (1977) is adopted;

$\dot{M}=-4 \times 10^{-13} \eta \frac{L / L_{\odot}}{g \cdot R / R_{\odot}} \quad\left(M_{\odot} \cdot \mathrm{yr}^{-1}\right)$,

or equivalently,

$$
\begin{aligned}
\log (-\dot{M})= & -4.67+\log \eta+1.5 \log \left(L / L_{\odot}\right) \\
& -\log \left(M / M_{\odot}\right)-2 \log T_{\mathrm{eff}}
\end{aligned}
$$

where $L, g, R, T_{\mathrm{eff}}$, and $M$ are stellar luminosity, surface gravity, radius, effective temperature, and initial mass, respectively. The mass loss efficiency parameter $\eta$ is assumed to be $1 / 3$ (Renzini \& Voli 1981). 

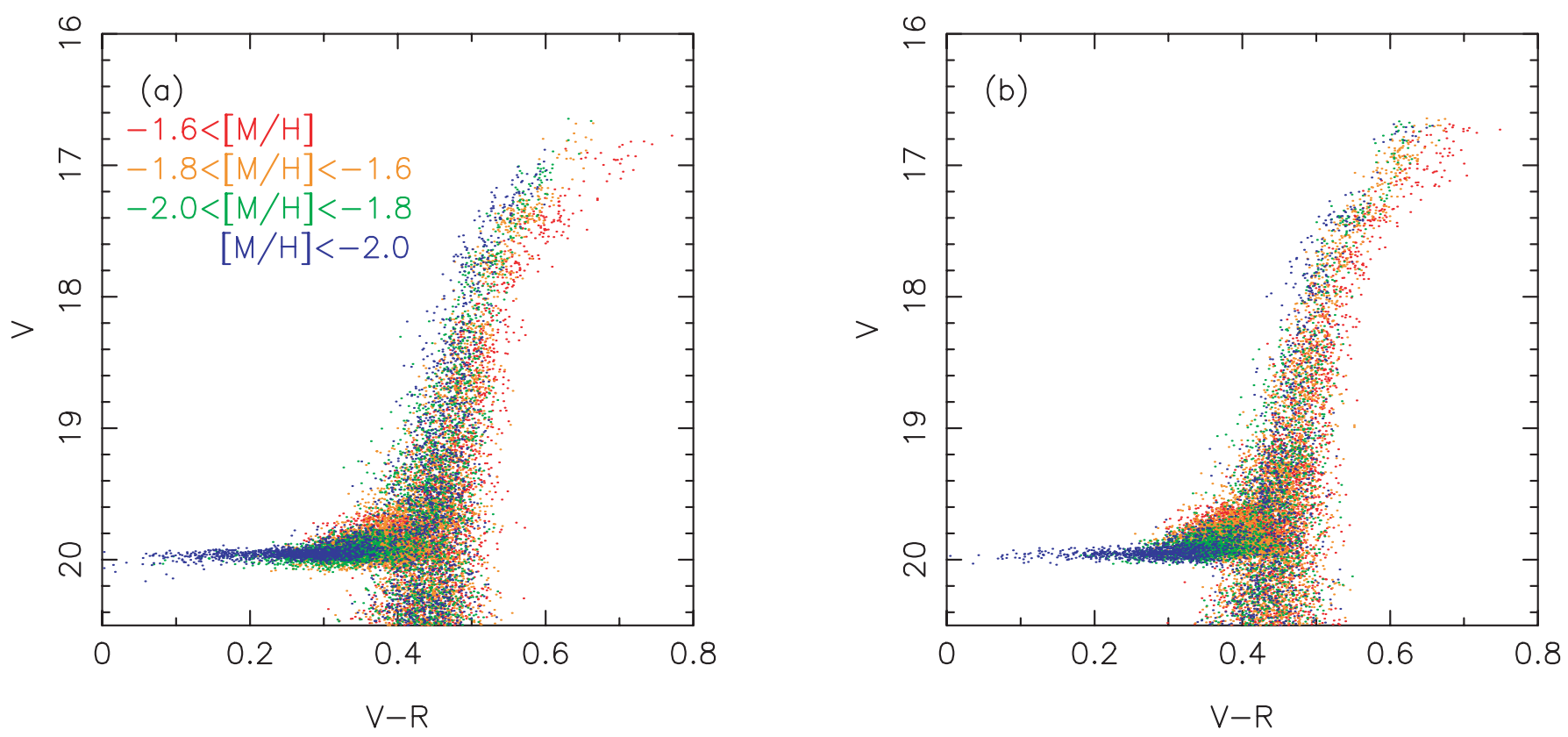

Fig. 5. Simulated CMDs of models B (panel a)) and C (panel b)). Adopted errors and data completeness are the same as those in Fig. 4, while the number of stars in each panel is increased for presentation purposes. Colours correspond to the following metallicity ranges; blue: $[\mathrm{M} / \mathrm{H}]<-2.0$; green: $-2.0 \leq[\mathrm{M} / \mathrm{H}]<-1.8$; orange: $1.8 \leq[\mathrm{M} / \mathrm{H}]<-1.6$; red:-1.6 $\leq[\mathrm{M} / \mathrm{H}]$.

The horizontal branch (HB) stars are distributed on the HR diagram according to a modified Gaussian mass distribution equation (Lee et al. 1990);

$$
\begin{aligned}
\Phi(M) \propto & {\left[M-\left(\overline{M_{\mathrm{HB}}}-\Delta M\right)\right]\left(M_{\mathrm{RGB}}-M\right) } \\
& \times \exp \left[-\frac{\left(\overline{M_{\mathrm{HB}}}-M\right)^{2}}{\sigma^{2}}\right],
\end{aligned}
$$

where $\sigma$ is a mass dispersion factor, and $\overline{M_{\mathrm{HB}}} \equiv\left(M_{\mathrm{RGB}}-\Delta M\right)$ is the mean mass of HB stars. A value of $\sigma=0.06 M_{\odot}$ has been chosen to represent the HB distributions similar to those of Galactic globular clusters (Rood 1973; Lee et al. 1990). This is equivalent to considering the statistical dispersions of mass loss rate along the RGB.

To convert the theoretical temperature-luminosity data to the observable colour-magnitude plane, a stellar spectral library by Lejuene et al. (1998) is used. This consists of Kurucz's (1992) spectra for hotter stars (O-K), Bessell et al.'s (1989, 1991) and Fruks et al.'s (1994) spectra for M giants, and Allard \& Hauschudt's (1995) for M dwarfs. In the original model spectra, systematic deviations become apparent when colourtemperature relations computed from the models are compared to the empirical ones at $1 Z_{\odot}$. The library adopted here is a version that the authors made by correcting the original library. The corrections are especially important for $\mathrm{M}$ star models. The fundamental parameters $\left(T_{\text {eff }}, g\right.$, and $\left.[\mathrm{M} / \mathrm{H}]=\log \left(Z / Z_{\odot}\right)\right)$ are wide enough to cover all spectral types and luminosity classes that appear in observed CMDs; $3 \times 10^{3} \leq T_{\text {eff }} \leq 5 \times 10^{4}$, $-1.02 \leq \log g \leq 5.5 ;-5.0 \leq[\mathrm{M} / \mathrm{H}] \leq 1.0$. To reduce computational time, synthetic magnitudes and colours tabulated by Lejuene et al. (1998) are linearly interpolated in $\log Z, \log T_{\text {eff }}$, and $\log g$.

\subsection{Comparison with the CMD of the Draco dSph}

In Sect. 2, models B and $\mathrm{C}$ are shown to fit well the observed trends in the $[\mathrm{Mg} / \mathrm{Fe}]-[\mathrm{Fe} / \mathrm{H}]$ diagram. Next, we discuss if the CMDs predicted by these models can reproduce the observed ones. Ideally, the SFH would be studied using a CMD which satisfies the following two conditions: (1) it covers a whole galaxy; (2) it is significantly deeper than the turnoff of the oldest stellar population. Unfortunately, such CMDs are not available for the galaxies discussed here. The situation, however, is better for the dSph Draco. Recently Piatek et al. (2001) imaged nine fields in and around the Draco dSph by using the KPNO $0.9 \mathrm{~m}$ telescope and presented the CMDs down to a luminosity level $\sim 2$ mag fainter than the HB. Thus, we mainly discuss the dSph Draco in this section and briefly mention the dSphs Sextans and Ursa Minor.

Figure 3 shows the observed CMD of the $\mathrm{C} 0$ field in the Draco dSph (Piatek et al. 2001), which is characterised by the narrow RGB and the well-populated red HB. The HB in Fig. 1 of Piatek et al. (2001) is somewhat distorted due to RR Lyrae stars which are marked with open circles in Fig. 3. Therefore, we do not take seriously the inconsistency between the observed and simulated blue HB morphology $(V-R<0.1)$. Historically, Baade \& Swope (1961) obtained the CMD of the Draco dSph and found that the RGB is generally similar to those of metal-poor globular clusters, although it is rather wide. The authors also found that the populous RHB is incompatible with the low metallicity of a Population II system. This is the notorious second-parameter problem of the dSph Draco.

Figures $4 \mathrm{a}$ and $4 \mathrm{~b}$ represent the CMDs simulated by models $B$ and $C$, respectively, where the galactic age is assumed to be 12 Gyr, which is similar ages of Galactic globular clusters (e.g., Carretta et al. 2000); values of other parameters are written in Table 1. The photometric errors and detection 


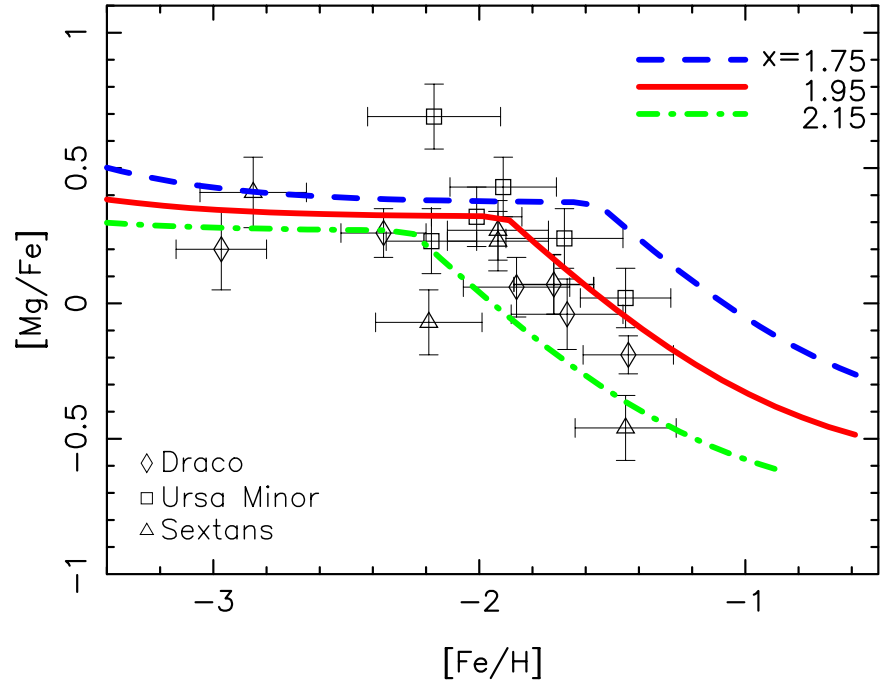

Fig. 6. The same as Fig. 1, but for models E-G. Different lines mean different IMF powers; model $\mathrm{E}$ ( $x=1.75$ : dashed line); model $\mathrm{F}$ ( $x=$ 1.95: solid line); model G ( $x=2.15$; dotted-dashed line). The star formation time scale is the same as for the solar neighbourhood disc, i.e., $\epsilon_{\mathrm{SF}}=0.2\left(\mathrm{Gyr}^{-1}\right)$. (This figure is available in color in electronic form.)

completeness in the simulations are taken from Piatek et al. (2001). Both models can reproduce characteristics of the CMD of the dSph Draco, such as narrow RGB and heavily populated RHB. Despite the metallicity dispersion $(-3 \leq[\mathrm{Fe} / \mathrm{H}] \leq$ $-1.4)$, the theoretical CMDs results in narrow RGBs. This is due to the so-called age-metallicity degeneracy as explained below.

Figure 5 shows CMDs simulated by assuming models B and $\mathrm{C}$, where different colours represent different metallicity range as described in the figure. For presentation purpose, Figs. 5a and $\mathrm{b}$ contain larger numbers of stars than those of Figs. 4a and b, respectively. The adopted observational conditions are the same as those of Fig. 4. Clearly, there is little correlation between the colour and metallicity of RGB stars because of the age-metallicity degeneracy. Since stars of higher metallicity are younger, the metallicity and age effects on the RGB colours are cancelled out. This keeps the RGB narrow and tight. The age effect appears in the HB morphology too. Since only metal-poor and old ( $>10 \mathrm{Gyr}$ ) stars can evolve to $\mathrm{BHB}$, younger core-He burning stars lie in the red part of the HB. The populous red HBs in Fig. 5 clearly show this age effect on the HB morphology. A weak correlation between the stellar colours and metallicities is found in the bright $\left(m_{V}<18\right.$ or $\left.M_{V}<-1\right)$ RGB.

In model $\mathrm{C}$, the RHB is more populous and brighter than in model B. This is because the longer duration of the star formation period increases the number of younger and more massive core-He burning stars. However, the difference between models $B$ and $C$ is so small that it is difficult to determine the final epoch of star formation from the CMD that does not reach down to the turnoff level. Nevertheless, it can be said that, in the dSph Draco, chemical evolution was very slow and the duration of star formation was at least longer than 3.9-6.5 Gyr.
This evolutionary picture can explain both the narrow RGB and the populous red HB of the Draco dSph. Thus, the second parameter problem in the $\mathrm{dSph}$ Draco is solved for the first time by introducing relatively long duration of star formation $(>3.9$ $6.5 \mathrm{Gyr}$ ) which is fully consistent with the observed abundance patterns.

\section{Discussion}

The abundance patterns and the CMDs of the dSphs Draco, Sextans, and Ursa Minor share the common features. It can be said that they have similar SFHs and CEHs. The CMD of the Sextans dSph (see Fig. 2 in Suntzeff et al. 1993; Fig. 1 in Shetrone et al. 2001) is characterised by a narrow RGB and a red HB similar to the dSph Draco. This suggests that the SFH of the dSph Sextans is similar to that of the dSph Draco. In the CMD of the Ursa Minor dSph (see Fig. 1 in Shetorne et al. 2001), the blue HB is more populous and the RGB is narrower. This might suggest that the time scale of star formation was shorter in the dSph Ursa Minor. Nevertheless, it can be said that, in the Draco dSph, the SFR was very low and the duration of star formation was at least longer than 3.9-6.5 Gyr. For the other dSphs, it is safe to say that the characteristics of the SFHs are the low SFRs and the long durations (>several Gyrs) of the star formation period.

For the dSph Draco, model C may conflict with the CMD obtained by the Hubble Space Telescope (Grillmair et al. 1998). Through a comparison with the fiducial lines of metal-poor globular clusters M 68 and M92, Grillmair et al. (1998) suggested that the dSph Draco is older than M 68 and M92 by $1.6 \pm 2.5$ Gyr. Their result, however, seems inconclusive. Firstly, the sparseness of the photometric sample makes it difficult to measure the contribution of any intermediate-age population. Although a predominant RHB is the characteristic of the dSph Draco, the few RHB stars appeared in their CMDs. Secondly, they used different techniques for photometry and presented the combined results. For bright stars aperture photometry was adopted, while the point spread function fitting method was used for faint stars. The border is unclear. This causes systematic discrepancies between the photometry of faint and bright stars. Third, a comparison with M 68 and M 92 is questionable. They used the $F 606 W$ filter of the WFPC2 system. However, the WFPC2 Instrument Handbook recommends that the $F 555 W$ filter is a better approximation to the Johnson $V$-band than the $F 606 \mathrm{~W}$. Their filter selection could have reduced the accuracy of the photometric calibration. Because of the combination of these uncertainties, it is dangerous to place much faith on their age estimation. More data are required to clarify these matters and images of a wide special coverage are particularly important.

In the above discussion, the decline in $[\mathrm{Mg} / \mathrm{Fe}]$ observed in the dSphs is interpreted as the effect of the chemical enrichment by SNeIa. Since the signal-to-noise ratios of the data (Shetrone et al. 2001; $S / N \simeq$ 13-36) for the dSphs are lower than other observations for metal-poor stars (e.g., McWilliams et al. 1995; $S / N \simeq 30-40$ ), one might argue that the quality of the data is not high enough to study the CEHs. However, the goal of Shetrone et al. (2001) was the measurement of overall 


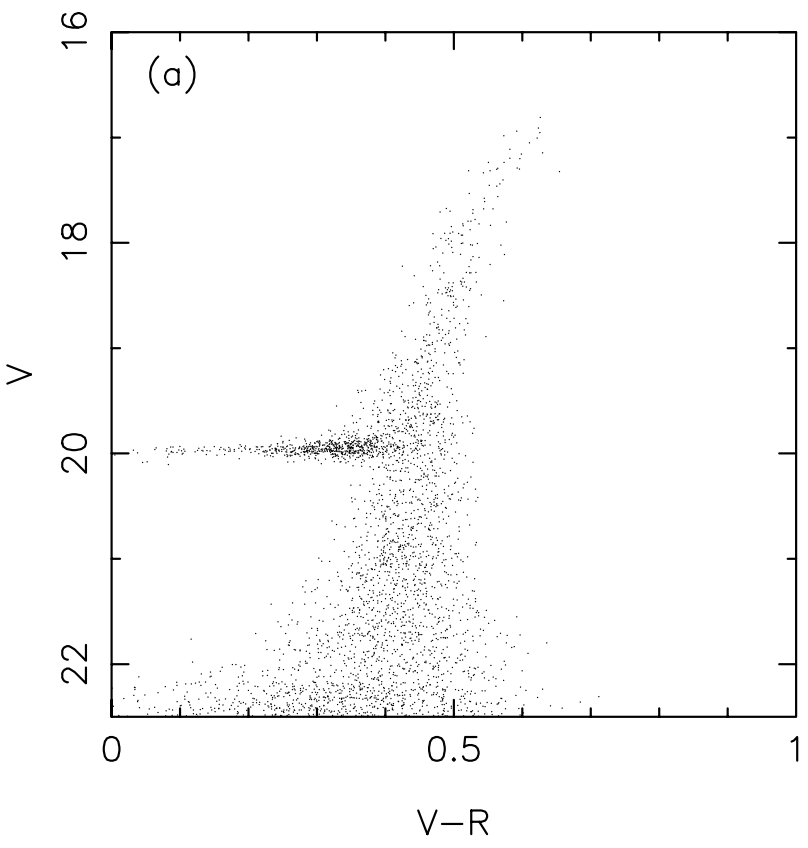

Fig. 7. The same as Fig. 4, but for models $\mathrm{F}$ and $\mathrm{G}$.

abundance differences, not absolute values as presented in their study, and it can be said that $[\alpha / \mathrm{Fe}]$ declines at $[\mathrm{Fe} / \mathrm{H}] \simeq-2$ on average (see Figs. 4 and 5 in Shetrone et al. 2001). Thus, it is a pertinent interpretation that enrichment by SNeIa causes the decline of $[\alpha / \mathrm{Fe}]$. Spectroscopic observations of high signal-tonoise ratios are required to confirm this.

We have shown that low SFRs can explain the observed trends in the $[\mathrm{Mg} / \mathrm{Fe}]-[\mathrm{Fe} / \mathrm{H}]$ diagram based on models assuming the Salpeter IMF. However, models of steeper IMFs and higher $\epsilon_{\mathrm{SF}}$ can also reproduce the trends. The steeper IMFs lead to slow chemical evolution, so that SNe Ia start to explode at low metallicity. This results in the decline of $[\mathrm{Mg} / \mathrm{Fe}]$.

Figure 6 shows chemical evolution models E-G assuming different IMFs, where the IMF slopes are $x=1.75,1.95$, and 2.15 , respectively, and $\epsilon_{\mathrm{SF}}=0.2$ (a standard $S F R$ for the solar neighbourhood; Arimoto et al. 1992). These models, except for model E, are also consistent with the observations. As with model $\mathrm{A}$, the abundance ratios predicted by model $\mathrm{E}$ are too high and thus inconsistent with the observations. For the observed metallicity range $([\mathrm{Fe} / \mathrm{H}]<-1.4)$, the trends of $[\mathrm{Mg} / \mathrm{Fe}]$ of models $\mathrm{E}-\mathrm{G}$ are very similar to those of models A-D, although they become different at higher metallicity (see Fig. 1).

Figures $7 \mathrm{a}$ and $\mathrm{b}$ represent the CMDs simulated by models $\mathrm{F}$ and $\mathrm{G}$, respectively. The photometric errors and detection completeness are taken from Piatek et al. (2001), i.e., the same as the simulations of models $\mathrm{B}$ and $\mathrm{C}$ shown in Fig. 4. The CMDs in Fig. 7 resemble those in Fig. 4 in terms of the morphologies of RGB and HB. The slow chemical evolution and the long ( $>1.6 \mathrm{Gyr}$ ) durations of star formation of models $\mathrm{F}$ and $G$ result in a narrow RGB and the populous RHB by the same reasons as those of models $B$ and $C$. This indicates that both abundance patterns and morphologies of the RGB and HB

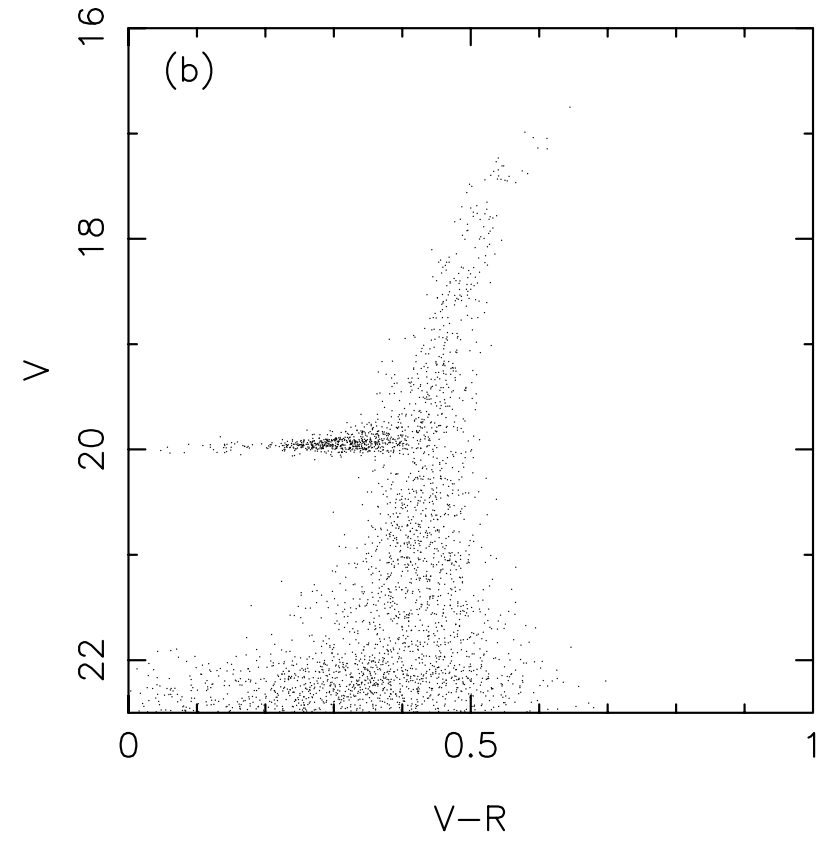

become similar when models have similar age-metallicity relations (AMRs).

Figures $8 \mathrm{a}$ and $8 \mathrm{~b}$ show the AMRs of models A-D and $E-G$, respectively. The figures demonstrate that the AMRs of models $E-G$ with the steeper IMFs result in very similar to those of models A-D with the lower SFRs at lowmetallicity $([\mathrm{Fe} / \mathrm{H}]<-1.4)$. Since both the abundance patterns and the morphologies of the RGB and HB are affected by $\mathrm{CEHs}$, an additional information is necessary to solve the degeneracy of IMF and SFR. In the models E-G the initial star formation lasts only 1.6-2.2 Gyrs, while in the models $\mathrm{B}-\mathrm{C}$ it continues as long as 3.9-6.5 Gyrs. Therefore, the models $\mathrm{B}-\mathrm{C}$ should give systematically brighter turnoff magnitudes than the models $\mathrm{E}-\mathrm{G}$, and magnitude difference between the main sequence turnoff and the $\mathrm{HB} V(\mathrm{TO}-\mathrm{HB})$ in the models $\mathrm{E}-\mathrm{G}$ should be smaller than in the models $\mathrm{B}-\mathrm{C}$. The models $\mathrm{E}-\mathrm{G}$ give $V(\mathrm{TO}-\mathrm{HB})$ is $\sim 3.1 \mathrm{mag}$, while the models $\mathrm{B}$ and $\mathrm{C}$ does $V(\mathrm{TO}-\mathrm{HB}) \sim 3.1 \mathrm{mag}$ and $\sim 2.8 \mathrm{mag}$, respectively. Deeper images with wider field of views will allow us to determine the final epochs of the initial star formation.

Figures 1 and 6 show that $[\mathrm{Mg} / \mathrm{Fe}]$ converges at higher metallicity in the models with the different SFRs and that it does not in those with the different IMFs. This difference allows us to derive the IMF and SFR independently. Abundance patterns in more massive dwarf galaxies and/or dwarf irregular galaxies will test whether the IMF or $S F R$ produces in the abundance patterns and low metallicities in the dSphs. We note that even steeper $(x>2.75)$ IMFs are required to explain the low metallicities of dwarf irregular galaxies if the IMF is the primary cause of the low metallicities. The CMD of the dwarf irregular galaxies in the Local Group reveal that they contain very old ( $>10 \mathrm{Gyr}$ ) populations as well as young ones, i.e., the star formation and chemical enrichment have continued for at least $10 \mathrm{Gyr}$. Since they are still metal-poor, extremely steep $(x>2.75)$ IMFs are required, which have never been 

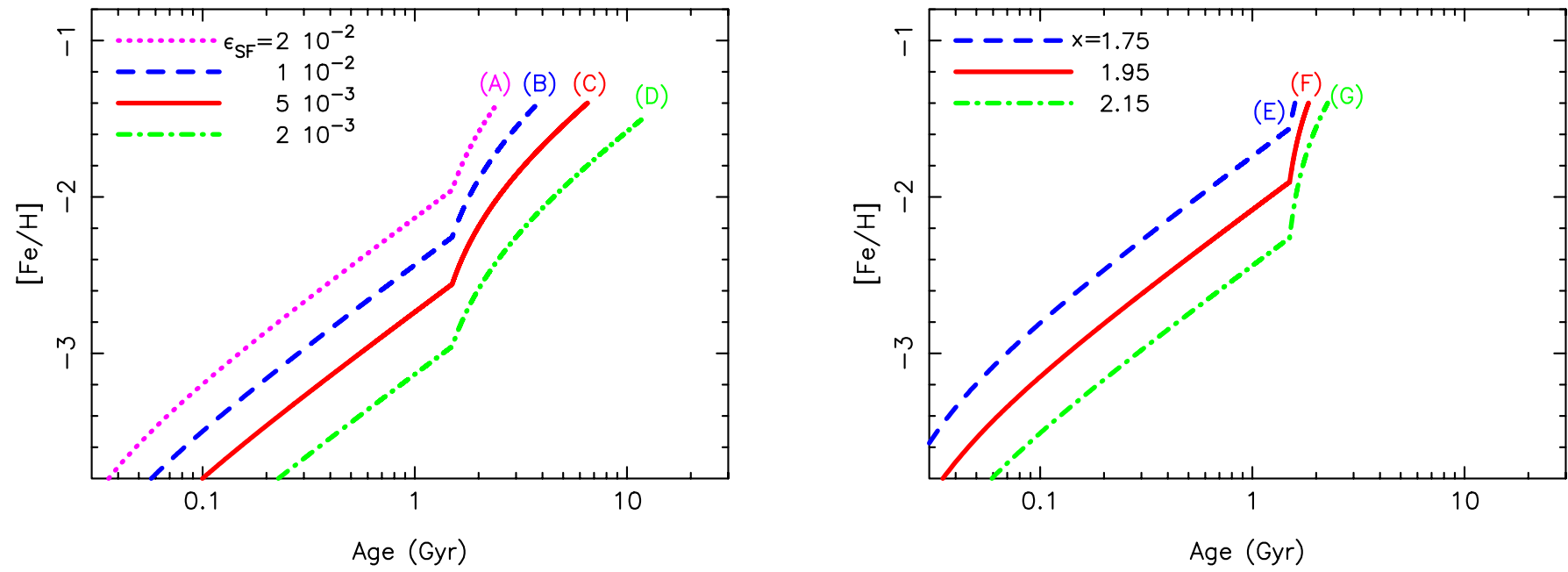

Fig. 8. Age-metallicity relations of models $A-D\left(\right.$ panel a)) and $E-G\left(\right.$ panel b)). The adopted values of $\epsilon_{\mathrm{SF}}\left(\mathrm{Gyr}^{-1}\right)$ or IMF power are shown on the panel. (This figure is available in color in electronic form.)

reported. Therefore, we believe that low SFRs are the primary characteristic of the SFHs of the dSphs.

So far, the gas infall and outflow during star formation have been neglected, since no evidence of the gas infall and outflow has presented. Metallicity distribution could provide a clue to judge whether gas infall or outflow should be considered. We stress that spectroscopic observations is indispensable to derive a metallicity distribution. Figure 5 demonstrates little correlation between the colours and metallicities of the RGB stars. Because of the age-metallicity degeneracy and contamination by AGB stars, a stellar colour is not always a good indicator of the metallicity. Thus, a careful procedure should be adopted to convert stellar colours into metallicities (Ikuta 2001), although a relatively simple technique is often used (e.g., Harris \& Harris 1999).

Long lasting star formation at a very low rate explains the observed trend of $[\mathrm{Mg} / \mathrm{Fe}]$ and the CMDs. Because of the low $S F R$ s, plenty of gas ( $\sim 97$ percent of the galaxy mass) still remain even at the final epoch of star formation (i.e., $t=\Delta T_{\mathrm{SF}}$ ). The remaining gas has to be removed to complete star formation and to evolve to a gas-poor system. Energy injection from supernovae could not be a sufficient mechanism to expel the gas from dwarf galaxies (MacLow \& Ferrara 1999; Ikuta 2001). If this is the case, gas removal from dwarf galaxies should result from external mechanisms such as ram pressure stripping and/or tidal shocks. Studying the SFHs of 18 dwarf galaxies in the Local Group based on the CMDs derived by a uniform method of photometry, Ikuta (2001) found that the durations of star formation period correlate with the distances from the Milky Way or M31. The correlation may suggest that the environmental effects play a key role in the evolution of the dwarf galaxies. Numerical simulations (Moore et al. 1998; Mayer et al. 2001) clearly showed that late-type dwarf galaxies entering the dark matter halo of a massive galaxy are transformed into early-type owing to repeated tidal stripping and dynamical instabilities. Thus, we conclude that the star formation in the dSphs Draco, Sextans, and Ursa Minor terminated due to gas stripping by the Milky Way.
The hierarchical clustering galaxy formation model suggested that star formation in dwarf galaxies can only occur before the cosmological re-ionisation epoch and there is no major star formation activities later because gas cannot cool and condense to form stars (e.g., Cen 2001). This appears to be inconsistent with stellar populations observed in the Local Group dwarf galaxies. CMDs obtained by the HST (e.g., Ikuta 2001) and our study presented in this paper clearly show extended and recent star formation activities in the Local Group dwarf galaxies.The issue is still being debated. However, a more recent simulation (Kitayama et al. 2001) suggested that star formation can occur in small objects if they have baryonic mass larger than the threshold mass of $10^{9} M_{\odot}$ and $10^{6} M_{\odot}$ at redshifts of $<3$ and $\sim 5$, respectively. The masses of today's dSphs in the Local Group are $10^{7}-10^{8} M_{\odot}$ (e.g., Mateo 1998) and our results imply that progenitors of today's dSphs lost $\sim 97 \%$ of their mass after the long lasting star formation. Therefore, the masses of the progenitors are estimated at $10^{9}-10^{10} M_{\odot}$. Since these exceed the threshold predicted by Kitayama et al. (2001), the SFHs derived here do not contradict recent simulations of the formation of dwarf galaxies.

\section{Conclusion}

Based on comparisons between the theoretical chemical evolution models and the observed abundance patterns, we conclude that the initial star formation continued for a long duration (>3.9-6.5 Gyr) in the dSphs Draco, Sextans, and Ursa Minor. Our simulation of the CMDs shows that the long duration of star formation can solve the second parameter problem of the Draco dSph. Because of the age-metallicity degeneracy, the RGBs are kept narrow and tight despite their large metallicity dispersions.

We have discussed the two cases which are consistent with the observed abundance patterns and the CMDs. The first case is a combination of low $\operatorname{SFRs}\left(\epsilon_{\mathrm{SF}}=1 \times 10^{-2}-5 \times 10^{-3} \mathrm{Gyr}^{-1}\right)$ and the Salpeter IMF $(x=1.35)$, while the second is a combination of the solar neighbourhood $S F R\left(\epsilon_{\mathrm{SF}}=0.2 \mathrm{Gyr}^{-1}\right)$ and 
the steeper IMFs ( $x=1.75-2.15)$. The two cases are discriminated neither by the abundance patterns nor by the CMDs of bright stars. However, the initial star formation period is as long as 3.9-6.5 Gyrs if the SFR is low, while it is much shorter (1.6$2.2 \mathrm{Gyrs}$ ) if the $S F R$ is high. Thus, turnoff magnitude should be different between the two cases. Deeper images with wider field of views allow us to determine the final epochs of the initial star formation.

Acknowledgements. We wish to express our gratitude to the anonymous referee for very helpful suggestions and comments. We are also grateful to T. Kodama, and H. Susa, for fruitful discussion and comments and to B. Jones who carefully read this manuscript and gave us helpful comments and suggestions. C.I. wishes to thank the Japan Society for Promotion of Science for financial support.

\section{References}

Allard, F., \& Hauschildt, P. H. 1995, ApJ, 445, 433

Aparicio, A., Gallart, C., Chiosi, C., \& Bertelli, G. 1996, ApJ, 469, L97

Arimoto, N., \& Yoshii, Y. 1986, A\&A, 164, 261

Arimoto, N., Yoshii, Y., \& Takahara, F. 1992, A\&A, 253, 21

Baade, W., \& Swope, H. H. 1961, AJ, 66, 300

Bertelli, G., Mateo, M., Chiosi, C., \& Bressan, A. 1992, ApJ, 388, 400

Bessel, M. S., Brett, J. M., Scholz, M., \& Wood, P. R. 1989, A\&AS, 77,1

Bessel, M. S., Brett, J. M., Scholz, M., \& Wood, P. R. 1991, A\&AS, 89,335

Blumenthal, G. R., Faber, S. M., Primack, J. R., \& Rees, M. J. 1994, Nature, 311, 517

Bressan, A., Fagotto, F., Bertelli, G., \& Chiosi, C. 1993, A\&AS, 100, 647

Carretta, E., Gratton, R. G., Clementini, G., \& Fusi Pecci, F. 2000, ApJ, 533, 215

Cen, R. 2001, ApJ, 549, L195

Chen, B. 1998, ApJ, 506, L79

Cole, S., Aragón-Salamanca, A., Frenk, C. S., Navarro, J. F., \& Zepf, S. E. 1994, MNRAS, 271, 781

Côté, P., Oke, J. B., \& Cohen, J. G. 1999, AJ, 118, 1645

Dolphin, A. 1997, New Astron., 2, 397

Edvardsson, B., Andersen, J., Gustafsson, B., et al. 1993, A\&AS, 102, 603

Fagotto, F., Bressan, A., Bertelli, G., \& Chiosi, C. 1994a, A\&AS, 104, 365

Fagotto, F., Bressan, A., Bertelli, G., \& Chiosi, C. 1994b, A\&AS, 105, 29

Ferraro, F. R., Fusi Pecci, F., Tosi, M., \& Buonanno, R. 1989, MNRAS, 225, 115

Fruks, M. A., Plez, B., Thé, P. S., et al. 1994, A\&AS, 105, 311

Gallart, C., Freedman, W. L., Aparicio, A., Bertelli, G., \& Chiosi, C. 1999, ApJ, 514, 665

Greggio, L., Marconi, G., Tosi, M., \& Focardi, P. 1993, AJ, 105, 894

Greggio, L., Tosi, M., Clampin, M., et al. 1999, ApJ, 504, 725

Girardi, L., Bressan, A., Chiosi, C., Bertelli, G., \& Nasi, E. 1996, A\&AS, 117, 113

Gratton, R. G., \& Sneden, C. 1987, A\&A, 178, 179

Grillmair, C. J., Mould, J. R., Holtzman, J. A., et al. 1998, AJ, 115, 144
Hachisu, I., Kato, M., Nomoto, K., \& Umeda, H. 1999, ApJ, 519, 314

Harris, G. L. H., \& Harris, W. E. 2000, AJ, 120, 2423

Hernandez, X., Valls-Gabaud, D., \& Gilmore, G. 1999, MNRAS, 304, 705

Hodge, P. W. 1971, ARA\&A, 9, 35

Ibata, R. A., Gilmore, G., \& Irwin, M. J. 1994, Nature, 370, 194

Iglesias, C. A., Rogers, F. J., \& Wilson, B. G. 1992, ApJ, 397, 717

Ikuta, C. 2001, Ph.D. Thesis, University of Tokyo

Kauffmann, G., White, S. D. M., \& Guiderdoni, B. 1993, MNRAS, 264, 201

Kitayama, T., Susa, H., Umemura, M., \& Ikeuchi, S. 2001, MNRAS, 326,1353

Kobayashi, C., Tsujimoto, T., Nomoto, K., Hachisu, I., \& Kato, M. 1998, ApJ, 503, L155

Kurutz, R. L. 1992, in The Stellar Populations of Galaxies, ed. B. Barbuy, \& A. Renzini (Dordrecht: Kluwer), 225

Lee, Y.-W., Demarque, P., \& Zinn, R. 1990, ApJ, 350, 155

Lejeune, Th., Cuisinier, F., \& Buser, R. 1998, A\&AS, 130, 65

MacLow, M.-M., \& Ferrara, A. 1999, ApJ, 513, 142

Mateo, M. L. 1998, ARA\&A, 36, 435

Matteucci, F., \& Brocato, E. 1990, ApJ, 365, 539

Matteucci, F., \& Greggio, L. 1986, A\&A, 154, 279

Mayer, L., Governato, F., Colpi, M., Moore, B., \& Quinn, T. 2001, ApJ, 547, L123

McWilliams, A., Preston, G. W., Sneden, C., \& Searle, L. 1995, AJ, 109, 2757

Moore, B., Lake, G., \& Katz, N. 1998, ApJ, 495, 139

Nomoto, K., Iwamoto, K., \& Kishimoto, N. 1997, Science, 276, 1378

Nomoto, K., Thielemann, F.-K., \& Yokoi, K. 1984, ApJ, 286, 644

Piatek, S., Pryor, C., Armandroff, T. E., \& Olszewski, E. W. 2001, AJ, 121,841

Reimers, D. 1977, A\&A, 61, 217

Renzini, A., \& Voli, M. 1981, A\&A, 94, 175

Rood, R. T. 1973, ApJ, 184, 815

Searle, L., \& Zinn, R. 1978, ApJ, 225, 357

Shetrone, M. D., Côté, P., \& Sargent, W. L. W. 2001, ApJ, 548, 592

Smecker-Hane, T. A., \& McWilliam, A. 1999, in Spectrophotometric Dating of Stars and Galaxies, ed. I. Hubeny, S. Heap, \& R. Cornett, ASP Conf. Proc., 192, 150

Smecker-Hane, T. A., Stetson, P. B., Hesser, J. E., \& Lehnert, M. D. 1996, in From Stars to Galaxies: The Impact of Stellar Physics on Galaxy Evolution, ed. C. Leitherer, U. Fritze-von Alvensleben, \& J. Huchra (ASP San Francisco), ASP Conf. Ser., 98, 328

Suntzeff, N. B., Mateo, M., Terndrup, D. M., et al. 1993, AJ, 418, 208

Thielemann, F.-K., Nomoto, K., \& Hashimoto, M. 1996, ApJ, 460, 408

Tinsley, B. M. 1980, Fund. Cosmic Phys., 5, 287

Tolstoy, E. 1996, ApJ, 462, 684

Tolstoy, E., \& Saha, A. 1996, ApJ, 462, 672

Tosi, M. 1991, AJ, 102, 951

Tsujimoto, T., Nomoto, K., Yoshii, Y., et al. 1996, MNRAS, 277, 945

Vader, J. P. 1986, ApJ, 305, 669

Vallenari, A., Chiosi, C., Bertelli, G., \& Ortolani, S. 1996, A\&A, 309, 358

White, S. D. M., \& Rees, M. J. 1978, MNRAS, 183, 341

Yanny, B., Newberg, H. J., Kent, S., et al. 2000, ApJ, 540, 825

Yoshii, Y., Tsujimoto, T., \& Nomoto, K. 1996, ApJ, 462, 266 